\title{
Análisis del sistema de medición en una fábrica ensambladora de electrónicos
}

\section{Analysis of the measurement system in an electronic assembly factory}

LÓPEZ-ACOSTA, Mauricioł, MONTOYA-CASTRO, Andrea, CHACARA-MONTES Allán y VELARDE-CANTÚ, José Manuel

Instituto Tecnológico de Sonora, Unidad Navojoa, México.

ID $1^{\mathrm{er}}$ Autor: Mauricio, López-Acosta / ORC ID: 0000-0003-3728-9576

ID $1^{\text {er }}$ Coautor: Andrea, Montoya-Castro

ID $2^{\text {do }}$ Coautor: Allán, Chacara-Montes / ORC ID: 0000-0002-0567-0017

ID $3^{\text {er }}$ Coautor: José Manuel, Velarde-Cantú / ORC ID: 0000-0002-1697-8551

DOI: $10.35429 /$ JTIP.2020.10.4.18.26

Recibido: Enero 20, 2020; Aceptado: Junio 30, 2020

\begin{abstract}
Resumen
La importancia del desarrollo de herramientas estadísticas en el campo laboral manufacturero parte de la lucha diaria de los procesos de producción industrial contra la variabilidad. El análisis del sistema de medición pretende evaluar la variabilidad asociada al método de medición, utilizado en un proceso productivo. Considerando el instrumento (Gage) y el operador que realiza la medición, para con ello poder identificar si se puede considerar como aceptable. El objetivo de la investigación es Evaluar la confiabilidad el sistema de medición utilizado en la línea de producción de candados electrónicos por medio de un estudio de Gage R\&R, para disminuir el número de piezas defectuosas. La línea de producción a evaluar, se dedica al ensamble y fabricación de candados electrónicos, cuenta con tres estaciones de inspección en las que se realizará un estudio de repetibilidad y reproducibilidad para obtener como resultado el porcentaje de exactitud asociado al desempeño de la máquina utilizada en el sistema de medición y en el personal. La metodología o procedimiento a seguir para el desarrollo del estudio de repetibilidad y reproducibilidad será llevado a cabo según lo mencionado por Pulido (2009), en los pasos descritos de un estudio R\&R corto y largo, del cual se tomaron los pasos más relevantes y aplicables según las características la presente problemática. La contribución del estudio es el desarrollo de un análisis del sistema de medición ofrece a la empresa el esquema de la situación actual de manera cuantitativa y cualitativa, y permite brindar una base sólida para el reconocimiento de oportunidades de mejora que puedan ayudar a la disminución de defectos reportados.
\end{abstract}

Variabilidad, Repetitividad, Reproducibilidad

\begin{abstract}
The importance of developing statistical tools in the manufacturing labor field starts from the daily struggle of industrial production processes against variability. The analysis of the measurement system aims to assess the variability associated with the measurement method used in the production process. Considering the instrument (Gage) and the operator that performs the measurement, in order to identify if it can be considered as acceptable. The objective of the research is to evaluate the reliability of the measurement system used in the production line of electronic locks through a Gage R\&R study, to reduce the number of defective parts. The production line under evaluation is dedicated to the assembly and manufacture of electronic padlocks, has three inspection stations in which a repeatability and reproducibility study is carried out to obtain as a result the percentage of accuracy associated with the performance of the machine in the measurement system and personnel. The methodology or procedure to be followed for the development of the repeatability and reproducibility study will be carried out as mentioned by Pulido (2009), in the described steps of a short and long $R \& R$ study, from which the most relevant and applicable steps were taken. according to the characteristics of the present problem. The contribution of the study is the development of an analysis of the measurement system offers the company the outline of the current situation in a quantitative and qualitative way and allows it to provide a solid basis for the recognition of improvement opportunities that can help to decrease reported defects.
\end{abstract}

Variability, Repeatability, Reproducibility

Citación: LÓPEZ-ACOSTA, Mauricio, MONTOYA-CASTRO, Andrea, CHACARA-MONTES Allán y VELARDECANTÚ, José Manuel. Análisis del sistema de medición en una fábrica ensambladora de electrónicos. Revista de Tecnologías en Procesos Industriales. 2020. 4-10:18-26.

$\dagger$ Investigador contribuido como primer autor. 


\section{Introducción}

En la actualidad, los métodos estadísticos son comprendidos como una herramienta valiosa para los estudios de sistemas de calidad. (Ortiz, 2016). Reconociendo el comercio globalizado y competitivo, las empresas buscan la satisfacción de sus clientes y la identificación de su marca en el mercado sobre a su competencia por medio de la calidad.

El ingeniero japonés Genichi Taguchi, da a conocer en sus aportaciones a la ingeniería de calidad, que el mayor enemigo de la calidad es la variabilidad. (Grima, 1995). Ofreciendo una respuesta oportuna a todo aquel que desee mejorar la calidad de sus procesos, productos o servicios para alcanzar sus objetivos como organización, y a su vez, delimitando las causas primordiales que no le permiten hacerlo, para con ello, dar solución y acercarse más al estado deseado.

Según Wheeler (2009), la reducción de variaciones en los procesos parte de la identificación de las fuentes, su cuantificación y el entendimiento de su sistema de medición.

De acuerdo con Fonseca (2018), con el propósito de dar evaluación a la influencia de los errores de medición de acuerdo con la calidad de las decisiones desarrolladas a partir de los datos ofrecidos por el mismo, es importante destacar a los estudios de análisis del sistema de medición. (Barbosa, 2014). Rodríguez, (2012) en su caso de estudio desarrolló un MSA aplicado a un brazo de un limpiaparabrisas, con un estudio de repetibilidad y reproducibilidad para los equipos de la planta en cuestión. Sus resultados mostraron un $32.62 \%$ de R\&R total y $26.99 \%$ de repetibilidad, por lo cual, se determina la causa de variación en la mayoría por el equipo utilizado para medir.

Desde otro ámbito, el MSA se ha utilizado para evaluar aparatos médicos o para el uso en el sector de salud. Ordorica, (2011) en su proyecto de "Validación de un sistema de medición aplicado a un estudio de termografía sensorial para la detección de desórdenes de traumas acumulados", lleva a cabo un estudio de R\&R para la validación de la termografía sensorial en seres humanos para asegurar la confiabilidad que tienen estos instrumentos de medición con el propósito de brindar información a futuras investigaciones.
Definición el problema, la empresa bajo estudio, se dedica a realizar ensambles electrónicos. Su principal problema se encuentra en la línea de candados electrónicos, la cual, afecta notablemente el rendimiento (Índice Yield), que son las piezas probadas pasadas a la primera (First PASS YIELD) que la empresa reporta. La situación que presenta en la organización demuestra la necesidad de llevar a cabo un estudio, para buscar la mejoría del rendimiento observado, ya que el indicador de desempeño presenta una baja considerable y una brecha identificada con la meta deseada por la organización, estando en un promedio de $93.4 \%$ comparado con el estado deseado de $97 \%$ de las piezas pasadas a la primera.

En base a lo anterior surge la necesidad de realizar las siguientes interrogantes:

¿El sistema de medición de la línea de producción de candados electrónicos es confiable?

b) ¿Cuáles son las causas principales de la variación presentada en las mediciones del proceso?

El Objetivo de investigación es: Evaluar la confiabilidad el sistema de medición utilizado en la línea de producción de candados electrónicos por medio de un estudio de Gage $\mathrm{R} \& \mathrm{R}$, para disminuir el número de piezas defectuosas.

\section{Metodología}

Sujeto Bajo Estudio: El estudio toma lugar en una fábrica ensambladora de electrónicos, una empresa grande con más de 900 empleados, con un giro industrial del sector secundario, donde sus principales productos incluyen alarmas de seguridad, alarmas de incendios, entre otros dispositivos de seguridad. Tomando como delimitación para el estudio los candados electrónicos que aquí se producen con el propósito de llevar a cabo un análisis del sistema de medición en la línea de producción, por lo cual es necesario contar con la información asociada al proceso de producción y el sistema de medición utilizado, así como la disponibilidad de la infraestructura necesaria, como lo son maquinaria de inspección y muestras de productos, para la implementación de un estudio de repetibilidad y reproducibilidad.

LÓPEZ-ACOSTA, Mauricio, MONTOYA-CASTRO, Andrea, CHACARA-MONTES Allán y VELARDE-CANTÚ, José Manuel. Análisis del sistema de medición en una fábrica ensambladora de electrónicos. Revista de Tecnologías en Procesos Industriales. 2020 
El procedimiento a seguir para el desarrollo del estudio de repetibilidad y reproducibilidad será llevado a cabo según lo mencionado por Pulido (2009), en los pasos descritos de un estudio R\&R corto y largo, del cual se tomaron los pasos más relevantes $\mathrm{y}$ aplicables según las características la presente problemática. A continuación, se describen los pasos a utilizar en este estudio:

Seleccionar operadores para conducir el estudio sobre el instrumento de medición dado.

Para comenzar el estudio se debe seleccionar la cantidad de operadores evaluando y tomando una decisión basada en la disponibilidad de mano de obra y la interacción del operador en el proceso de medición, ya que, esto puede significar un mayor número de operadores para el desarrollo del estudio con el objeto de obtener resultados confiables que muestren la variabilidad aportada por el operador al proceso. El número de operadores es seleccionado por el evaluador del estudio y debe ser mayor a dos. estudio.

Seleccionar piezas a utilizar en el

Se debe determinar el número de piezas a utilizar, el porcentaje de piezas aceptables y piezas no aceptables, y en caso de que el sistema a evaluar permita evaluar más de un tipo de piezas, elegir el modelo o tipo de pieza, mediante el análisis del porcentaje más alto de fallas de todos los modelos inspeccionados en esa prueba y seleccionar la cantidad necesaria de las mismas tomando en cuenta que para un estudio $R \& R$ se recomienda utilizar 10 piezas o más, sin embargo, esto puede variar dependiendo de si se desea realizar un estudio largo o corto. piezas.

Cálculo de repeticiones y etiquetado de

Es necesario calcular el número de repeticiones que realizará cada operador, el orden es aleatorio, en el cual las partes se dan a los operadores e identificar las piezas por aceptables y no aceptables, según Pulido (2019).

Identificar el método utilizado para medir y crear tabla de mediciones aleatorias.

\section{Recopilación de datos.}

Se realiza el estudio y se capturan los datos obtenidos en la tabla diseñada en el paso anterior.

\section{Análisis de resultados}

Se lleva a cabo el análisis estadístico de los datos para conocer el porcentaje de error presentado por el sistema de medición para con base a ello brindar una base para toma de decisiones $y$ conclusiones.

\section{Materiales}

Para el desarrollo de esta investigación es necesario contar con software de Excel para el manejo de la información y el software Minitab 18. Cabe mencionar que el estudio puede realizarse sin la necesidad del software Minitab 18, sin embargo, para beneficio de esta investigación, será utilizado para el concentrado, análisis y desarrollo de gráficas de los resultados del estudio.

\section{Resultados}

Para la prueba funcional se realizaron los siguientes pasos:

Seleccionar operadores para conducir el estudio sobre el instrumento de medición dado. Se seleccionaron a dos operadores para la realización del estudio. Esto basado en la disponibilidad de la mano de obra y considerando un mínimo de dos operadores para el cálculo de la reproducibilidad de las mediciones. De lo cual, se seleccionaron a los dos operadores que comúnmente operan dicha medición, que cuentan con más de un año operando en la empresa en la línea de producción bajo estudio y conocen el producto.

$$
\text { estudio. }
$$

$$
\text { Seleccionar piezas a utilizar en el }
$$

Se seleccionaron cuatro piezas a evaluar en el estudio, conociendo a dos de esas piezas como Aceptables o Buenas (Piezas Golden o de referencia) y el resto como Rechazadas o Malas. Determinando esta cantidad debido a la disponibilidad de las piezas, en cuestión de piezas que se encontraran como rechazadas $\mathrm{y}$ buenas del proceso. 
Cálculo de repeticiones y etiquetado de piezas.

El número de repeticiones se determinó basado en la poca disponibilidad de tiempo para realizar el estudio, y la cantidad de Slots por máquina. De lo cual, se estableció el mínimo de repeticiones a realizar, que es dos, para cubrir las pruebas necesarias en el tiempo disponible. Esto considerando que la prueba Funcional consta de cuatro máquinas con cuatro posiciones que deben evaluarse de manera individual. El número de repeticiones se determinó por medio del siguiente cálculo:

Numero de repeticiones $=($ Tiempo disponible de la maquina) / ((Piezas a evaluar) * (Tiempo de la prueba $) *($ Número de operadores $))=(30 \mathrm{~min}) /$ $((4) *(1 \mathrm{~min}) *(2))=3.75 \approx 4$

Se consideraron cuatro factores del contexto para el estudio: Tiempo disponible de la máquina, Piezas a evaluar, tiempo que dura la prueba y el número de operadores a realizar la prueba, para en base a la disponibilidad y el mínimo de dos repeticiones requeridas para el estudio, se determinó una cantidad de 4 repeticiones.

A su vez, se realizó el etiquetado de piezas categorizando de la siguiente manera: piezas 1 y 2 son Aceptables/Buenas, y piezas 3 y 4 son Rechazadas/Malas.

Identificar el método utilizado para medir y crear tabla de mediciones aleatorias.

Se identificó el tipo de mediciones como mediciones por atributos y se utilizó Minitab para crear el orden aleatorio de las piezas para crear la tabla de trabajo a utilizar para realizar el estudio

\section{Recopilación de datos.}

Se llevó a cabo el llenado de datos del estudio en las hojas de trabajo correspondientes, utilizando una hoja de trabajo por posición (Slot) en la máquina. Se realizó en cuatro máquinas de prueba Funcional con las que cuenta la línea de producción. Se prueba el número de pieza en la máquina según el orden aleatorio dado por la tabla y se compara con el estándar.
El código de error y su descripción, se obtiene cuando la máquina considera la pieza como Malo o No aceptable, esto indica en que fallo la pieza durante el proceso de medición.

Los resultados obtenidos del desarrollo del estudio de repetibilidad y reproducibilidad en la prueba Funcional 2 en el Slot 1 mostró un porcentaje de exactitud general del $68.8 \%$, de lo cual se puede identificar una tasa de error general del $31.3 \%$, de lo cual un $37.5 \%$ de piezas buenas fueron calificadas como malas y un $25 \%$ de piezas malas fueron calificadas como buenas y un 37.5 fueron el mismo elemento calificado de ambas maneras. Con un porcentaje de exactitud para el evaluador 1 del $75 \%$ y para el evaluador 2 del $62.5 \%$.

De acuerdo con el informe de exactitud determinado por la prueba realizada en Funcional 2 en el Slot 1, se muestra un porcentaje de exactitud más grande para el evaluador 1, a su vez, una mayor exactitud para las piezas malas que para las piezas buenas, como también un mayor porcentaje de exactitud en el ensayo 2. Obteniendo un mayor porcentaje de exactitud el evaluador 1 que el evaluador dos al momento de probar las piezas categorizadas como buenas, y un porcentaje de exactitud igual al momento de probar piezas categorizadas como malas.

Por otro lado, la clasificación incorrecta durante el estudio mostro una tasa de error del $31.3 \%$, donde destacó el elemento 2 al probar piezas buenas que fueron calificadas como malas $\mathrm{y}$ el elemento 4 al probar piezas malas calificadas como buenas. Donde se muestra una mayor tendencia del evaluador 2 a calificar piezas buenas como malas y una tendencia igual por parte de los evaluadores para calificar piezas malas como buenas, sin embargo, el evaluador 1 presenta un mayor porcentaje de piezas calificadas de ambas maneras.

Las pruebas se fueron repitiendo de la misma manera en las tres máquinas faltantes obteniendo como resultado: Los porcentajes de exactitud por máquina se calcularon por medio del promedio de los porcentajes de exactitud de sus Slots. Es decir, la suma del Promedio de exactitud de Slot 1, 2, 3, 4 y su división entre el número de Slots, en este caso igual a 4 . Los resultados fueron: Funcional $2=60.95 \%$, Funcional $4=53.15 \%$, Funcional $7=70.35 \%$, Funcional $10=53.15 \%$. 


\section{Prueba Prepot}

Seleccionar operadores para conducir el estudio sobre el instrumento de medición dado.

Se seleccionaron a dos operadores para la realización del estudio y la consideración de la diferencia en la variación presentada en el desempeño de los mismos. Esto basado en la disponibilidad de la mano de obra y considerando un mínimo de dos operadores para el cálculo de la reproducibilidad de las mediciones. estudio.

Seleccionar piezas a utilizar en el

Se seleccionaron cinco piezas a evaluar en el estudio, conociendo a tres de esas piezas como Aceptables o Buenas y el resto como Rechazadas o Malas.

Cálculo de repeticiones y etiquetado de piezas.

El número de repeticiones se determinó basado en la disponibilidad de tiempo para realizar el estudio. De lo cual, se establecieron tres repeticiones para la obtención de resultados más confiables ya que se contaba con tiempo disponible considerable para la realización del estudio. Esto considerando que la prueba Prepot consta de dos máquinas que deben evaluarse de manera individual.

Numero de repeticiones $=($ Tiempo disponible de la maquina ) / ((Piezas a evaluar) * (Tiempo de la prueba $)^{*}($ Número de operadores $)=(30 \mathrm{~min}) /$ $((5) *(1 \mathrm{~min}) *(2))=3$

A su vez, se realizó el etiquetado de piezas categorizando de la siguiente manera: piezas 1, 2 y 3 son Aceptables/Buenas, y piezas 4 y 5 son Rechazadas/Malas.

Identificar el método utilizado para medir y crear tabla de mediciones aleatorias.

Se identificó el método de medición utilizado como medición por atributos en criterios Pasa/Nopasa, por lo cual, se realizó el orden aleatorio de las piezas por medio de la herramienta de MSA por atributos de Minitab 18.
Los resultados obtenidos del desarrollo del estudio de repetibilidad y reproducibilidad en la prueba Prepot 1 mostró un porcentaje de exactitud general del $90 \%$, de lo cual se puede identificar una tasa de error general del $10 \%$, de lo cual un $16.7 \%$ de piezas buenas fueron calificadas como malas y un $0 \%$ de piezas malas fueron calificadas como buenas y un $30 \%$ fueron el mismo elemento calificado de ambas maneras. Con un porcentaje de exactitud para el evaluador 1 del $93.3 \%$ y para el evaluador 2 del $86.7 \%$.

De acuerdo al informe de exactitud determinado por la prueba realizada en Prepot 1 , se muestra un porcentaje de exactitud mayor para el evaluador 1, a su vez, una mayor exactitud para las piezas malas que para las piezas buenas, con un porcentaje de exactitud mayor en el ensayo 1 y 2. Obteniendo un porcentaje de exactitud mayor para el evaluador 1 al momento de probar las piezas categorizadas como buenas, y un porcentaje igual para ambos evaluadores al momento de calificar piezas categorizadas como malas.

Por otro lado, la clasificación incorrecta durante el estudio mostro una tasa de error del $10 \%$, donde destacó el elemento 3 siendo una pieza buena calificada como mala. Donde se muestra un porcentaje mayor del evaluador 2 a calificar piezas buenas como malas, y por calificar las mismas piezas de ambas maneras.

Se realizaron las mediciones para las dos máquinas siguientes. Se identificó un porcentaje de exactitud del sistema de medición en la prueba Prepot, en la cual se cuenta con dos máquinas de medición, con un $90 \%$ en la máquina 1 y un $86.7 \%$ en la máquina 2 . Con una mayoría de fallas de piezas buenas calificadas como malas.

\section{Prueba Final}

Seleccionar operadores para conducir el estudio sobre el instrumento de medición dado. Se seleccionaron a dos operadores para la realización del estudio y la consideración de la diferencia en la variación presentada en el desempeño de estos.

Seleccionar piezas a utilizar en el estudio. Se seleccionaron diez piezas a evaluar en el estudio, conociendo a siete de esas piezas como Aceptables o Buenas y el resto como Rechazadas o Malas. 
Cálculo de repeticiones y etiquetado de piezas.

Número de repeticiones $=($ Tiempo disponible de la maquina $) /(($ Piezas a evaluar) * (Tiempo de la prueba $) *($ Número de operadores $)=(60 \mathrm{~min}) /$ $((10) *(1 \min ) *(2))=3$

A su vez, se realizó el etiquetado de piezas categorizando de la siguiente manera: piezas 1, 2, 3, 5, 6, 8, 10 son Aceptables/Buenas, y piezas 4, 7 y 9 son Rechazadas/Malas.

Identificar el método utilizado para medir y crear tabla de mediciones aleatorias.

Los resultados evidencian el porcentaje de exactitud de cada una de las máquinas de la prueba Final de la siguiente manera: Final 1= $60 \%$, Final $2=55 \%$, Final $3=70 \%$, Final $5=$ $68.3 \%$. Teniendo las máquinas 1,3 y 5 una tendencia hacia la calificación de las piezas malas como aceptables o buenas, y la máquina 2 calificando lo aceptable o bueno como malo.

\section{Resultados generales}

Los resultados obtenidos por el sistema de medición utilizado en la línea de producción de candados electrónicos se presentaron en un rango del $53 \%$ al $90 \%$ de exactitud de medición (Ver Figura 1).

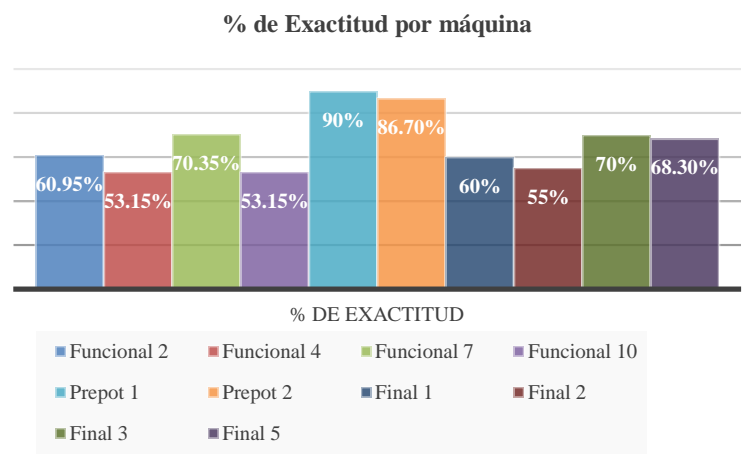

Figura 1 Porcentajes de exactitud de máquinas de prueba de inspección en Estudio R\&R

Fuente: (Elaboración propia)

Las principales causas de las fallas identificadas procedentes del sesgo de error en los estudios, considerando solo las fallas ocurridas en piezas Aceptables/Buenas, fueron las siguientes (Ver Figura 2):

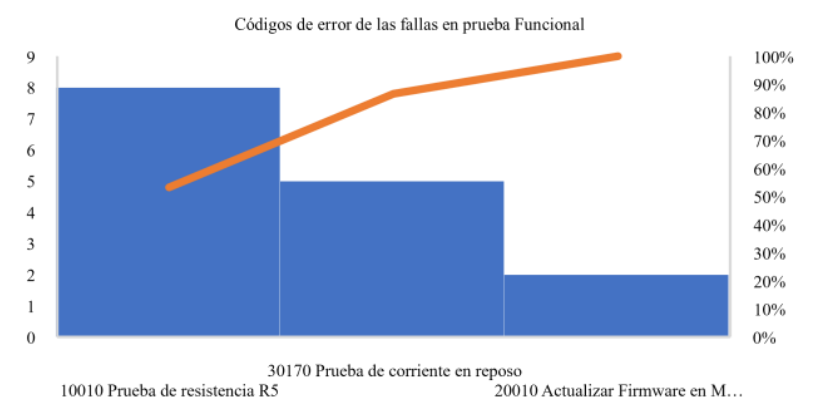

Figura 2 Códigos de error de las fallas prueba Funcional Fuente: (Elaboración propia)

A su vez, la prueba Prepot presentó un solo tipo de falla en su sesgo de error con piezas buenas calificadas como malas, siendo el código de error 30030 Verificar el voltaje de la batería.

\section{Conclusiones}

Los resultados obtenidos del análisis del sistema de medición aplicado en la línea de producción de candados electrónicos por medio del desarrollo de un estudio de repetibilidad y reproducibilidad permitieron cumplir con el objetivo planteado al conocer el porcentaje de exactitud de cada una de las máquinas de medición involucradas en la inspección del producto. El estudio de repetibilidad y reproducibilidad permite evaluar la confiabilidad de la máquina y la variación atribuida al usuario $\mathrm{u}$ operador de la prueba. La prueba se desarrolló en las tres pruebas de inspección que incluye el proceso de producción, identificados como pruebas por atributos con los criterios Bueno/Malo o Pasa/Nopasa, obteniendo como resultado el porcentaje de exactitud correspondiente, los cuales, se comportaron de la siguiente manera: Prueba Funcional 2=60.95\%, Funcional $4=53.15 \%$, Funcional $7=70.35 \%$, Funcional $10=$ $53.15 \%$, Prepot $1=90 \%$, Prepot $2=86.7 \%$, Final $1=60 \%$, Final $2=55 \%$, Final $3=70 \%$, Final $5=$ $68.3 \%$.

Dichos porcentajes son comparados a los rangos de aceptación de un sistema de medición según el Manual de MSA, donde un valor por debajo del $95 \%$ se considera como No Aceptable $\mathrm{y}$ un valor por arriba de $95 \%$ se considera Aceptable. Con ello, se llegó a concluir que los equipos de medición utilizados en la línea de producción de candados electrónicos No son Aceptables. Reconociendo también por parte de los operadores una variación importante $\mathrm{y}$ considerada como fuera de lo aceptable. 


\section{Recomendaciones}

De acuerdo con los resultados y conclusiones obtenidas en la presente investigación se extienden las siguientes recomendaciones para la toma de decisiones de acuerdo al panorama actual evaluado. Considerando los resultados No aceptables del estudio de repetibilidad y reproducibilidad se recomienda el ataque de los principales códigos de error presentados durante la prueba y la búsqueda de sus causas raíces para la implementación de acciones correctivas que le permitan a la empresa escalar hacia su meta establecida. Así como a su vez, de acuerdo a los resultados de baja aceptabilidad de la variación entre operadores, se recomienda la constante capacitación del personal, su verificación y una menor rotación del mismo para minimizar los efectos de la curva de aprendizaje en la variación aportada al proceso. Por otro lado, se recomienda analizar los límites establecidos en las pruebas de inspección de acuerdo con el diseño del producto, ya que, índices de exactitud bajos en todos los operadores, como se presentó en los resultados del presente estudio, puede significar un error en la definición de los límites de la prueba.

Mucho del trabajo de administrar un sistema de medición es dirigido al monitoreo y control de la variación, ((AIAG), 2010); Por lo cual, se recomienda llevar a cabo seguimiento de los resultados obtenidos por medio del desarrollo de otro estudio de repetibilidad y reproducibilidad en un periodo de 3 a 6 meses para la creación de una comparativa entre el estado actual y el estado con futuro y el uso de gráficos de control para el seguimiento del desempeño del gage, así como también, el desarrollo del mismo análisis para otras líneas de producción en las cuales la empresa presente la misma situación.

\section{Referencias}

Minitab. (2017). Análisis de sistemas de medición. Recuperado el 05 de Marzo de 2019, de http://support.minitab.com/es$\mathrm{mx} / \mathrm{minitab} / 17 /$ topic-library/qualitytools/measurement-system-analysis/gage-r-ranalyses/perform-gage-r-r-with-multipleoperators-and-one-part/
AIAG), A. I. (2010). Mesarument Systems Analysis Reference Manual (Vol. 4th Edition). Avila, Y. Y. (2013). Implementación de control estadístico de procesos para el control de la calidad y la mejora continua en una industria minera. Toluca, Estado de México, México. Recuperado el 12 de Marzo de 2019, de http://ri.uaemex.mx/handle/20.500.11799/1381 5

Barbosa, R. (2017). Monitoreo y análisis estadístico de procesos con aplicaciones. Universidad del Norte. Recuperado el 09 de Marzo de 2019, de https://books.google.com.mx/books?id=DNJC DwAAQBAJ\&dq=Control+estadistico+de+pro cesos\&source=gbs_navlinks_s

Ciro, M. B. (2011). Estadística básica aplicada. Ecoe Ediciones. Obtenido de https://books.google.es/books?id=tKQwDgAA QBAJ\&dq=estadistica+industrial \&lr=\&hl=es\& source=gbs_navlinks_s

G.F. Barbosa, G. P. (2014). R\&R (repeatability and reproducibility) gage study applied on gaps' measurement of aircraft assemblies made by a laser technology device. German Academic Society for Production Engineering (WGP). Recuperado el 11 de Febrero de 2019, de https://link-springer-

com.itson.idm.oclc.org/content/pdf/10.1007\%2 Fs11740-014-0553-z.pdf

Group, S. C. (6 de Noviembre de 2012). MSA Análisis del sistema de medición. Recuperado el 02 de Marzo de 2019, de https://spcgroup.com.mx/msa/

Héctor Quevedo Urias, B. R. (2014). Estadística para Ingeniería y Ciencias. Grupo Editorial Patria. Obtenido de https://books.google.com.mx/books?id=jvLhB AAAQBAJ\&dq=estadistica+industrial \&hl=es\& source=gbs_navlinks_s

Humberto Gutiérrez Pulido, R. d. (2009). Control Estadístico de Calidad y Seis Sigma. Guadalajara, México: McGRAWHill/INTERAMERICANA EDITORES, S.A DE C.V.

Jameel Haleel, A. H. (2018). Gage Repeatability and Reproducibility Study. Association of Arab Universities Journal of Engineering Sciences. 
Javier Ordorica Villalvazo, C. C. (2011). Validación de un sistema de medición aplicado a un estudio de termografía sensorial para la detección de desórdenes de traumas acumulados. Universidad Autónoma de Baja California.

Maldonado, R. M. (2018). Control Estadístico de la Calidad. Grupo Editorial Patria. Recuperado el 09 de Marzo de 2019, de https://books.google.com.mx/books?id=yImDwAAQBAJ\&dq=Control+estadistico $+d e+p$ rocesos\&source=gbs_navlinks_s

Manteiga, M. T. (2012). Estadística aplicada: Una visión instrumental. Ediciones Díaz de Santos. Obtenido de https://books.google.com.mx/books?id=8tocM TUkICkC\&dq=estadistica\&hl=es\&source=gbs_ navlinks_s

Manuel Rodríguez Medina, M. I. (2012). Análisis y mejoramiento del proceso de medición: Caso de estudio. Instituto Tecnológico de Ciudad Juárez. Juárez: Academia Journals. Recuperado el 08 de Febrero de 2019

Merli, G. O. (2012). Gestión de la Calidad: Control Estadístico y Seis Sigma. Revista de Estudios Interdisciplinarios en Ciencias Sociales.

Mikel Harry, P. S. (2010). The practitioner's guide to statistics and lean six sigma for process improvements. John Wiley \& Sons. Recuperado el 11 de Marzo de 2019, de https://books.google.com.mx/books?id=Crqm2 AmECD0C\&dq $=$ gage $+r \% 26 r \& h l=e s \&$ source $=$ gbs_navlinks_s

Molina, J. S. (2016). Estudio de repetibilidad y reproducibilidad (GR\&R). Universidad Autónoma del Estado de México. Obtenido de http://ri.uaemex.mx/bitstream/handle/20.500.11 799/64058/secme-

37772.pdf? sequence $=1 \&$ is Allowed $=y$

Ortiz, Ó. C. (2016). Sistema de gestión de calidad: Teoría y práctica bajo la norma ISO 2015. Ecoe Ediciones. Recuperado el 12 de Febrero de 2019, de https://books.google.com.mx/books?id=baUwD gAAQBAJ\&dq=sistemas + de + calidad\&source $=$ gbs_navlinks_s
Pandiripalli, B. (2010). Repeatability and Reproducibility Studies: A Comparison of Techniques. Wichita State University, Industrial and Manufacturing Engineering. Chaitanya Bharathi Institute of Technology. Recuperado el 11 de Febrero de 2019

Pedrera, C. H. (2016). Aplicación del control estadístico de procesos (CEP) en el control de su calidad. Santiago, Cuba.: Universidad de Oriente. Obtenido de http://web.b.ebscohost.com.itson.idm.oclc.org/e host/pdfviewer/pdfviewer?vid=4\&sid=391c 254 1-1547-4c03-b9a8bd9fcf604435\%40sessionmgr102

Pedro Grima Cintas, J. T.-M. (1995). Técnicas para la gestión de la calidad. Ediciones Díaz de Santos.

Rodolfo Gabriel Jasso, I. J. (2014). Aplicación de la metodología seis sigma para disminuir la variación de medición de la carga del resorte de fricción. Universidad Autónoma de Ciudad Juárez. Juárez: ResearchGate. Obtenido de https://www.researchgate.net/publication/29299 4535

Rufe, P. D. (2013). Fundamentals of Manufacturing, Third Edition. Society of Manufacturing Engineers. Recuperado el 05 de Febrero de 2019, de https://books.google.com.mx/books?id=Xs2Wx $787 \mathrm{hpcC} \& \mathrm{dq}=$ gage $+\mathrm{r}+\% 26+\mathrm{r} \&$ source=gbs_na vlinks_s

Teng, Z. J. (2002). A study on measurement system and its effect on process quality. Tianjin University. People's Republic of China. Recuperado el 11 de Febrero de 2019, de https://search-proquest-

com.itson.idm.oclc.org/pqdtglobal/docview/102 4997895/F88A6E22299E4227PQ/1 ?accountid= 31361

Vázquez, E. J. (2003). Seis-Sigma: metodología y técnicas. Editorial Limusa. Obtenido de https://books.google.com.mx/books?id=viHl0ib n9c4C\&dq=estudio+de+repetibilidad+y+reprod ucibilidad\&source $=$ gbs_navlinks_s

Wheeler, D. J. (2009). An Honest Gauge R\&R Study. SPC Ink. 
Yoel Portuondo Paisan, J. P. (2010). La repetibilidad y reproducibilidad en el aseguramiento de la calidad de los procesos de medición. Facultad de Ingeniería Mecánica, Universidad de Oriente. Obtenido de file:///C:/Users/andrea/Downloads/994-3560-1PB.pdf

Zurro, A. M. (2010). Compendio de atención primaria. Elsevier España. Recuperado el 11 de Marzo de 2019, de https://books.google.com.mx/books?id=JRqXq Yw2rAAC\&dq=estudio+de+repetibilidad $+y+r$ eproducibilidad\&source=gbs_navlinks_s. 